\title{
Seroprevalence of Bovine Brucellosis in Ethiopia: Systematic Review and Meta-Analysis
}

This article was published in the following Dove Press journal:

Veterinary Medicine: Research and Reports

\author{
Abebe Tesfaye' \\ Haileyesus Dejene $\mathbb{D}^{2}$ \\ Bemrew Admassu' \\ Takele Adugna Kassegn ${ }^{3}$ \\ Destaw Asfaw ${ }^{4}$ \\ Gashaw Getaneh Dagnaw' \\ Abebe Belete Bitew ${ }^{2}$ \\ 'Unit of Biomedical Science, College of \\ Veterinary Medicine and Animal Science, \\ University of Gondar, Gondar, Ethiopia; \\ ${ }^{2}$ Department of Veterinary Epidemiology \\ and Public Health, College of Veterinary \\ Medicine and Animal Science, University of \\ Gondar, Gondar, Ethiopia; ${ }^{3}$ Department of \\ Clinical Medicine, College of Veterinary \\ Medicine and Animal Science, University of \\ Gondar, Gondar, Ethiopia; ${ }^{4}$ Department of \\ Paraclinical Studies, College of Veterinary \\ Medicine and Animal Science, University of \\ Gondar, Gondar, Ethiopia
}

Correspondence: Haileyesus Dejene Email hailedejene64@gmail.com

\begin{abstract}
Brucellosis is an infectious and widespread zoonotic disease caused by bacteria of the genus Brucella and can induce considerable human suffering and huge economic losses in animals. Thus, the aim of this study was to conduct a systematic review and meta-analysis on the seroprevalence of bovine brucellosis in Ethiopia. PubMed, Science Direct, African Journals Online, and Google Scholar were used to search the articles. All references were screened and articles, which reported seroprevalence of bovine brucellosis in Ethiopia were included in the study. Meta-analysis using random-effects models was made to calculate the pooled seroprevalence of brucellosis. This review included 15 papers. The estimated pooled seroprevalence of brucellosis was found to be 3.0\% (95\% CI: 2.0, 4.0). The subgroup analysis showed that there was a statistically significant association between the disease and geographical location, setting, laboratory technique employed and study years. Also, there was some evidence of publication bias (Egger's test, $p=0.0003$ ) on studies reporting the prevalence of brucellosis in Ethiopia. This review proves a high seroprevalence of brucellosis in the country and appropriate control strategies are recommended. Moreover, further study on the risk factors is also required to develop cost-effective preventive strategies.
\end{abstract}

Keywords: brucellosis, cattle, Ethiopia, seroprevalence, meta-analysis

\section{Background}

Brucellosis, which is an infectious disease caused by gram-negative bacteria of the genus Brucella, is a widespread zoonosis disease that can induce considerable human suffering and huge economic losses in animals. ${ }^{1-3}$ Bovine brucellosis is usually caused by $B$. abortus. ${ }^{4,5}$ However, it has occasionally been caused by $B$. melitensis and $B$. suis in some instances where mixed farming is practiced. ${ }^{5}$

Even though brucellosis in livestock has been eradicated in industrialized countries, it is one of the most prevalent zoonotic diseases worldwide $^{6}$ with approximately 500,000 new cases of human infection every year, ${ }^{3}$ especially, in low-income tropical countries where agriculture is the mainstay. In this part of the world, the disease is endemic and neglected, with huge health and livelihood burdens. This is due to lack of effective control and proper disease surveillance. $^{7,8}$ In many developing countries like Ethiopia, brucellosis remains endemic and continues to be a major public and animal health problem. ${ }^{9}$ Brucellosis has been noted as one of the important livestock diseases in the country as it has been depicted the first report of brucellosis in the 1970s in Ethiopia ${ }^{10}$ and its reported seroprevalence is ranging from $1.5 \%$ to $22.6 \%$ were reported in the country. ${ }^{1,11}$ The disease impairs socio-economic development for livestock owners 
by causing significant loss of productivity through abortion, prolonged calving, kidding, or lambing interval, low herd fertility, and comparatively low milk production in farm animals.

Even though, most reports have made either limited geographic coverage or are relatively confined to a single agro-ecology, these stated evidences strongly suggest that bovine brucellosis might be a widespread problem in Ethiopia. ${ }^{7,11-14}$ Compiling the results of different studies fragmented by area and time is very important to show the disease burden at the country level within the specified period of time. Moreover, a comprehensive understanding of the disease occurrence in cattle across the country should be identified as this helps to shape possible future intervention programmes. Thus, the objective of this systematic review and meta-analysis was to estimate the seroprevalence of bovine brucellosis for evidence-based disease control in Ethiopia.

\section{Methods}

\section{Study Protocols}

The study was performed according to the Preferred Reporting Items for Systematic review and Meta-Analysis (PRISMA) guideline (Supplementary Figure 1). ${ }^{1,15} 1$ The checklist was used to make sure the inclusion of relevant information in the analysis. The outcome of interest was the proportion and odds ratio of seropositive for brucellosis. The study protocol had been registered on PROSPERO with reference number ID: CRD42020171243 and Available from: https://www.crd.york.ac.uk/prospero/dis play record.php? ID=CRD42020171243.

\section{Literature Search Strategy}

This systematic review and meta-analysis were aimed to determine the weighted seroprevalence of brucellosis. We retrieved published articles from various English electronic databases, including: PubMed/PubMed Central, Science Direct, Scopus, African Journals Online, and Google scholar until February 29, 2020. Article search was made following the medical subject heading (MeSH) terms. The key search terms were: "Brucellosis" OR "Brucella" AND "Seroprevalence" OR "Prevalence" OR "Seroepidemiology" AND "Risk factors" OR "Potential factors" AND "Cattle" OR "Bovine" AND "Ethiopia". We used "OR" and "AND" Boolean operators to identify studies with any of the keywords in their titles, abstracts and full texts. Moreover, unpublished thesis manuscripts were also accessed from various Ethiopian Universities and research centers.

\section{Inclusion and Exclusion Criteria}

We used the following inclusion criteria to confirm the eligibility of the searched papers: 1) original peerreviewed research articles and thesis conducted in Ethiopia; 2) cross-sectional studies that reported the seroprevalence of brucellosis; 3) studies with full texts; 4) targeted study population included bovine within any of the management system (intensive or extensive); in this context intensively managed bovine are those cattle which are kept indoor for whole day or that only be out of the house for only few hour in a day for recreation whereas extensively managed bovine are cattle that are kept on the grazing pasture and get their feed by grazing with or without supplementation in the early morning and late afternoon; 5) studies were performed using serological diagnostic tests RBPT for screening and CFT or ELISA for confirmation; 6) studies provided the total sample size and the outcome of interest (number of positive samples); 7) studies published in English language; and 8) studies published online between 2009 up to February 29, 2020. Papers which did not meet the above-mentioned criteria were excluded. Besides, the references of the selected papers were checked manually to find relevant papers that were not retrieved in the database search.

\section{Study Selection and Data Extraction}

Records identified from various electronic databases, indexing services and directories were exported to MENDELEY reference manager software version 1.19.5 (Mendeley Ltd.) with compatible formats. Duplicate records were identified, documented and removed with Mendeley. Some duplicates were addressed manually due to variation in reference styles across sources. Thereafter, two authors (HD and AT) independently screened the title and abstracts with predefined inclusion criteria. Two authors (HD and AT) also independently collected fulltexts and evaluated the eligibility of them for final inclusion. In each case, the rest authors played a critical role in solving discrepancies arose between two authors to come up to consensus.

Similarly, data extraction format was prepared based on first author, publication year, study year, geographical location (region and zone), study design, sampling method, sample size, diagnostic test, setting, number of positive samples, and their corresponding negative cases 
among the study groups (Data Supplement). Seroprevalence of brucellosis was calculated by dividing the number of positive cases by the total number of individuals used for the study in a given population at a given period. The study effect size and their corresponding confidence intervals were calculated from the extracted data. Study searching strategies and exclusion criteria are presented in detail in Supplementary Figure 1. Mendeley version 1.19.5 was used to catalogue the initial literature search results and to manage citations. Microsoft Excel datasheet was used to code and manage all extracted information from all relevant studies.

\section{Study Quality Assessment}

Two independent researchers were evaluated the quality of the included papers using a quality assessment checklist (standard strengthening the Reporting of Observational Studies in Epidemiology checklist (STROBE)) (Supplementary Check List 1). This quality assessment checklist includes 22 items constituting various sections of the articles such as title, abstract, introduction, methods, results, and discussion. The checklist included items assessing objectives, different components of the methods (eg, study design, sample size, study population, bias, statistical methods), results, limitations, and funding of the studies. The assigned scores were determined from 0 to 44 . Following the checklist (STROBE), searched papers were classified into 3 groups: low quality score $(<15.5)$, moderate quality (15.5-29.5), and high quality: (30.0-44.0). ${ }^{16,17}$ The quality of the included studies was indicated in Supplementary Table 1 .

\section{Meta-Analysis}

Data on the seroprevalence and corresponding $95 \%$ confidence intervals (CIs) of the disease were calculated for each study. The pooled prevalence estimate was computed using the formula given by Barendregt et al. ${ }^{18}$ Forest plot diagram was employed to present the variations among studies, outcomes of meta-analysis that display estimates of the seroprevalence, and their corresponding CIs of all included studies together with the pooled effect size. Similarly, subgroup analyses for the primary outcome (seroprevalence of brucellosis) will be done by study region, laboratory technique employed (CFT or ELISA) and setting. Cochran's Q-statistics and inverse variance index $\left(I^{2}\right)$ were computed to determine the heterogeneity and inconsistency (true variation) among studies, respectively. Similarly, we considered the $I^{2}$ values of 25,50 , and
$75 \%$ as low, medium and high heterogeneity, respectively. ${ }^{19}$ The tau statistics $\left(\tau^{2}\right)$ was used to assess the variance of the effect size estimates across the population of the study. Galbraith plot diagram was also constructed to assess the heterogeneities of study level estimates. Based on the heterogeneity assessment result, we used Der Simonian and Laird's random-effects method (if the $p$-value of the $\mathrm{Q}$ test was $<0.05$ and $I^{2}$ was $>50 \%$ ) or Mantel-Haenszel's fixed-effects method to pool the estimations. $^{20}$ Small study effects and publication bias presence were then visualized using funnel plot diagrams and, Egger's and Begg's asymmetry tests. ${ }^{21}$ A funnel plot was computed using the logarithm of effect size and its corresponding standard error of the effect size. STATA software version 16 (Stata Corp., College Station, TX, USA) was used to do the meta-analysis.

\section{Result \\ Search Result}

A total of 134 potentially relevant studies were identified from several sources including PubMed/PubMed Central, Science Direct, Scopus, African Journals Online, and Google scholar. From these, 20 duplicated articles were removed with the help of MENDELEY and manual tracing. The remaining 114 records were screened using their titles and abstracts and 85 of them were excluded. Full texts of 29 records were then evaluated for eligibility. From these, 14 articles were excluded due to the outcome of interest was found missing, insufficient and/or ambiguous. Finally, a total of 15 articles fulfilled the eligibility criteria and quality assessment and thus included for systematic review and meta-analysis (Supplementary Figure 1).

\section{Study Characteristics}

The 15 eligible studies which were considered for determining the seroprevalence of brucellosis in cattle are summarized in Supplementary Table 1..$^{4,7,11-14,22-30}$ The studies were published in the year between 2009 and 2019. All the selected studies were cross-sectional study design in nature (Supplementary Table 1).

\section{Publication Bias and Small Study Effect Assessment}

We assessed bias and small study effects by funnel plot observation and Egger's test for small study effects. The result of effect estimates against its standard error showed that there was some evidence of publication bias on 
studies reporting the seroprevalence of brucellosis in Ethiopia (Egger's test, $p=0.0003$ ) (Supplementary Figure 2).

\section{Meta-Analysis}

Due to the expected variation between studies, randomeffects meta-analyses were carried out using the total sample size and number of positives (effect size and standard error of the effect size). The meta-analysis indicated that between-study variability was high $\left(\mathrm{T}^{2}=0.00 ; \mathrm{I}^{2}=\right.$ 96.3\%, Q-test $=377.8, d f=14$ and $P<0.001)$. Individual study prevalence estimates ranged from $0.0 \%$ to $11 \%$ with the overall random pooled prevalence of $3 \%$ (95\% CI: 2.0 , 4.0). Studies weighted approximately equal with weights on individual studies ranging from $1.7 \%$ to $8.0 \%$ due to high heterogeneity between studies. Supplementary Figure 2 presents the forest plot derived from the meta-analysis (Supplementary Figure 3).

\section{Subgroup Meta-Analysis}

Subgroup analyses were done for study locations (central, eastern, northern, western, southern and selected areas of Ethiopia) (Supplementary Table 2), setting (farm and at field) (Supplementary Table 3), laboratory technique employed (CFT and ELISA) (Supplementary Table 4) and year of study (study year between 2009-2011, 2012-2015 and 2016-2018) (Supplementary Table 5). Thus, high seroprevalence was observed in Southern Ethiopia 8\% (95\% CI: 4.0, 12.0) followed by Northern Ethiopia, 3\% (95\% CI: 1.0, 7.0), whereas the least prevalence was observed in Central Ethiopia 1\% (95\% CI: 0.0, 3.0) and Eastern Ethiopia 1\% (95\% CI: 1.0, 3.0\%) (Supplementary Table 2). Similarly, the pooled seroprevalence of bovine brucellosis from the different settings: at fields and farms was $4 \%(95 \%$ CI: 3.0, 6.0) and $1 \%(95 \%$ CI: $0.0,2.0$ ), respectively (Supplementary Table 3). Also, the prevalence of bovine brucellosis from the different laboratory technique employed: CFT and ELISA was 3\% (95\% CI: $2.0,4.0)$ and 1\% (95\% CI: 1.0, 3.0), respectively (Supplementary Table 4). Between the different study years, the seroprevalence of bovine brucellosis was $4 \%$ (95\% CI: $3.0,5.0), 2 \%(95 \%$ CI: $1.0,3.0)$ and $2 \%(95 \%$ CI: $0.0,4.0)$ in studies conducted between 2009-2011, 2012-2015 and 206-2018, respectively (Supplementary Table 5).

\section{Meta-Regression}

Meta-regression analysis was done for each variable included in the study separately. The variables included were setting, study year as categorical variable, study location and laboratory technique employed. Those variables with p-values $<0.1$ were used in the multivariable meta-regression analysis. Study location and setting had significant value and retained in the final multivariable analysis. Results of final multivariable meta-regression are summarized in Supplementary Table 6.

\section{Discussion}

Brucellosis is a widespread zoonotic disease that can induce considerable human suffering and huge economic losses in animals. ${ }^{1-3}$ It has a significant public health implication for a pastoral community in consequence of lifestyles, feeding habits, close contact with animals, low awareness, and poor hygienic conditions which favors infection. ${ }^{1,11}$ Also, it can generally cause significant loss of productivity through abortion, prolonged calving, kidding, or lambing interval, low herd fertility, and comparatively low milk production in farm animals. The disease impairs socio-economic development for livestock owners, which represents a vulnerable sector in rural populations in general and pastoral communities in particular. Even though, most reports have made either limited geographic coverage or are relatively confined to a single agroecology, these stated evidences strongly suggest that brucellosis might be a widespread problem in Ethiopia., ${ }^{71-14}$

Out of 15 original studies with 15,562 serum samples included in this study, the pooled seroprevalence of bovine brucellosis was 3.0\%. This result is in concordance with the previous meta-analysis report from dairy cattle in Ethiopia where the pooled prevalence was $3.3 \%$. Similarly, the current finding was similar with the reports of who reported prevalence of $3.4 \%$ from cattle selected in different areas in Cameron. However, the current finding was higher than the reports of ${ }^{31}$ and $^{32}$ who reported a pooled prevalence of $1.9 \%$ from dairy cattle in China and $0.001 \%$ in Iran, respectively. Also, the current finding was higher when compared with the report in Uganda. ${ }^{33}$ Meanwhile, the current finding was lower than the reports in Zimbabwe, Ghana, Pakistan, Jordan, Cameroon, Tanzania and India. ${ }^{5,34-39}$ Moreover, among the different countries, China, India and Iran are practiced different control strategies of bovine brucellosis ${ }^{3,31,32}$ however, the other countries did not practice control strategies. ${ }^{3-39}$ The difference in the seroprevalence of bovine brucellosis in the different studies could be due to differences in the geographical location and farming system between the different studies. Therefore, information on the actual seroprevalence of the disease in the country 
help the policymakers to develop appropriate strategies regarding prevention and control protocols.

In the present study, the subgroup analysis showed that there was a statistically significant association between the disease and geographical location, setting, laboratory technique employed and study years. Also, there was evidence of publication bias and small study effects (Egger's test, $\mathrm{p}=0.0003$ ) on studies reporting the seroprevalence of bovine brucellosis in Ethiopia.

\section{Conclusion and Recommendations}

This systematic and meta-analysis has certain limitations, including: limited and/or lack of studies in many regions of the country and the findings were heterogeneous. In conclusion, the results of this meta-analysis show that the pooled prevalence estimate of the disease in the country is $3.0 \%$. Thus, by considering the significance of bovine brucellosis in the national economy, strategies to reduce the prevalence and burden of brucellosis should be prioritized and offered adequate funding.

\section{Data Sharing Statement}

All data generated during this study are included as supplementary information.

\section{Acknowledgments}

We thank the authors of the literature.

\section{Author Contributions}

All authors made substantial contributions to conception and design, acquisition of data, or analysis and interpretation of data; took part in drafting the article or revising it critically for important intellectual content; agreed to submit to the current journal; gave final approval of the version to be published; and agree to be accountable for all aspects of the work.

\section{Disclosure}

The authors declare that they have no competing interests.

\section{References}

1. Migisha R, Nyehangane D, Boum Y, et al. Prevalence and risk factors of brucellosis among febrile patients attending a community hospital in south western Uganda. Sci Rep. 2018;8(1):1-8. doi:10.1038/s41598018-33915-9

2. Carvalho AV, Mol JPS, Xavier MN, Paixão TA, Lage AP, Santos RL. Pathogenesis of bovine brucellosis. Vet J. 2010;184(2):146-155. doi:10.1016/j.tvj1.2009.04.010

3. Mufinda FC, Boinas F, Nunes C. Prevalence and factors associated with human brucellosis in livestock professionals. Rev Saude Publica. 2017;51:1-10. doi:10.1590/s1518-8787.2017051006051
4. Tschopp R, Bekele S, Moti T, Young D, Aseffa A. Brucellosis and bovine tuberculosis prevalence in livestock from pastoralist communities adjacent to Awash National Park, Ethiopia. Prev Vet Med. 2015;120(2):187-194. doi:10.1016/j.prevetmed.2015.03.004

5. Ndukum JA, Moctar M, Mouiche M, Houli NB, Ngwa VN. Seroprevalence and associated risk factors of brucellosis among indigenous cattle in the Adamawa and north regions of Cameroon seroprevalence and associated risk factors of brucellosis among indigenous cattle in the Adamawa and north regions of Cameroon. Vet Med Int. 2018;2018:1-10. doi:10.1155/2018/3468596

6. Corbel MJ. Brucellosis: an overview. Emerg Infect Dis. 1997;3 (2):213-221. doi:10.3201/eid0302.970219

7. Terefe Y, Girma S, Mekonnen N, Asrade B. Brucellosis and associated risk factors in dairy cattle of eastern Ethiopia. Trop Anim Health Prod. 2017;49(3):599-606. doi:10.1007/s11250-017-1242-7

8. Mcdermott J, Grace D, Zinsstag J. Economics of brucellosis impact and control in low-income countries framework for the economic assessment of brucellosis. Rev Sci Tech. 2013;32(1):249-261. doi:10.20506/rst.32.1.2197

9. Asmare K, Krontveit RI, Ayelet G, Sibhat B, Godfroid J, Skjerve E. Meta-analysis of brucella seroprevalence in dairy cattle of Ethiopia. Trop Anim Health Prod. 2014;46(8):1341-1350. doi:10.1007/s11250014-0669-3

10. Domenech J. Enquête sérologique sur la brucellose du dromadaire en Ethiopie. Rev Elev Med Vet Pays Trop. 1977;30(2):141-142. doi:10.19182/remvt.8090

11. Asmare K, Sibhat B, Molla W, et al. The status of bovine brucellosis in Ethiopia with special emphasis on exotic and cross bred cattle in dairy and breeding farms. Acta Trop. 2013;126(3):186-192. doi:10.1016/j.actatropica.2013.02.015

12. Megersa B, Biffa D, Abunna F, Regassa A, Godfroid J, Skjerve E. Seroepidemiological study of livestock brucellosis in a pastoral region. Epidemiol Infect. 2012;140(5):887-896. doi:10.1017/ S0950268811001178

13. Mekonnen H, Kalayou S, Kyule M. Serological survey of bovine brucellosis in barka and arado breeds (Bos indicus) of Western Tigray, Ethiopia. Prev Vet Med. 2010;94(1-2):28-35. doi:10.1016/j. prevetmed.2009.12.001

14. Jergefa T, Kelay B, Bekana M, Teshale S, Gustafson H, Kindahl H. Epidemiological study of bovine brucellosis in three agro-ecological areas of central Oromiya, Ethiopia. OIE Rev Sci Tech. 2009;28 (3):933-943. doi:10.20506/rst.28.3.1939

15. Moher D, Shamseer L, Clarke M, et al. Preferred reporting items for systematic review and meta-analysis protocols (PRISMA-P) 2015 statement. Syst Rev. 2015;4(1):1-9. doi:10.1186/2046-4053-4-1

16. Von Elm E, Douglas G, Egger M, Pocock S, Gotzsche P, Vandenbroucke $J$. The strengthening the reporting of observational studies in epidemiology (STROBE) statement: guidelines for reporting observational studies. Ann Intern Med. 2007;147(8):573-578. doi:10.7326/0003-4819-147-8-200710160-00010

17. Moher D, Liberati A, Tetzlaff J, Altman DG, Prisma T. Preferred reporting items for systematic reviews and meta-analyses: the PRISMA statement. Int J Surg. 2010;8(5):336-341. doi:10.1016/j. ijsu.2010.02.007

18. Barendregt JJ, Doi SA, Lee YY, Norman RE, Vos T. Meta-analysis of prevalence. J Epidemiol Community Health. 2013:1-5.

19. Higgins JPT, Thompson SG. Quantifying heterogeneity in a meta-analysis. Stat Med. 2002;21(11):1539-1558. doi:10.1002/ sim. 1186

20. Tufanaru C, Munn Z, Stephenson M, Aromataris E. Fixed or random effects meta-analysis? Common methodological issues in systematic reviews of effectiveness. Int $J$ Evid Based Healthc. 2015;13 (3):196-207. doi:10.1097/XEB.000000000000065

21. Borenstein M, Hedges LV, Higgins JPT, Rothstein HR. Introduction to Meta-Analysis. Chichester, U.K.: John Wiley and Sons, Ltd., Chichester, U.K.; 2009:277-291. 
22. Tschoop R, Birhanu A, Sabi Yao S, et al. Bovine tuberculosis and brucellosis prevalence in cattle from selected milk cooperatives in Arsi zone, Oromia region, Ethiopia. BMC Vet Res. 2013;9.

23. Megersa B, Biffa D, Niguse F, Rufael T, Asmare K, Skjerve E. Cattle brucellosis in traditional livestock husbandry practice in Southern and Eastern Ethiopia, and its zoonotic implication. Acta Vet Scand. 2011;53(1):1-8. doi:10.1186/1751-0147-53-24

24. Tesfaye G, Tsegaye W, Chanie M, Abinet F. Seroprevalence and associated risk factors of bovine brucellosis in Addis Ababa dairy farms. Trop Anim Health Prod. 2011;43(5):1001-1005. doi:10.1007/ s11250-011-9798-0

25. Megersa B, Biffa D, Abunna F, Regassa A, Godfroid J, Skjerve E. Seroprevalence of brucellosis and its contribution to abortion in cattle, camel, and goat kept under pastoral management in Borana, Ethiopia. Trop Anim Health Prod. 2011;43(3):651-656. doi:10.1007/ s11250-010-9748-2

26. Edao BM, Hailegebreal G, Berg S, et al. Brucellosis in the Addis Ababa dairy cattle: the myths and the realities. BMC Vet Res. 2018;14 (1):1-9. doi:10.1186/s12917-018-1709-4

27. Adugna KE, Agga GE, Zewde G. Seroepidemiological survey of bovine brucellosis in cattle under a traditional production system in western Ethiopia. OIE Rev Sci Tech. 2013;32(2):765-773. doi:10.20506/rst.32.2.2218

28. Ibrahim N, Belihu K, Lobago F, Bekana M. Sero-prevalence of bovine brucellosis and its risk factors in Jimma zone of Oromia Region, South-western Ethiopia. Trop Anim Health Prod. 2010;42 (1):35-40. doi:10.1007/s11250-009-9382-z

29. Dirar BG, Nasinyama GW, Gelalcha BD. Seroprevalence and risk factors for brucellosis in cattle in selected districts of Jimma zone, Ethiopia. Trop Anim Health Prod. 2015;47(8):1615-1619. doi:10.1007/s11250-015-0910-8

30. Chaka H, Aboset G, Garoma A, Gumi B, Thys E. Cross-sectional survey of brucellosis and associated risk factors in the livestockwildlife interface area of Nechisar National Park, Ethiopia. Trop Anim Health Prod. 2018;50(5):1041-1049. doi:10.1007/s11250-018$1528-4$
31. Ran X, Cheng J, Wang M, et al. Brucellosis seroprevalence in dairy cattle in China during 2008-2018: a systematic review and metaanalysis. Acta Trop. 2018.

32. Mirnejad R, Jazi FM, Mostafaei S, Sedighi M. Epidemiology of brucellosis in Iran: a comprehensive systematic review and meta-analysis study. Microb Pathog. 2017;109:239-247. doi:10.1016/j.micpath.2017.06.005

33. Nguna J, Dione M, Apamaku M, et al. Seropositivity in cattle, goats and humans in Iganga District, Uganda. Pan Afr Med J. 2019;8688:1-10.

34. Matope G, Bhebhe E, Muma JB. Seroprevalence of brucellosis and its associated risk factors in cattle from smallholder dairy farms in Zimbabwe. Trop Anim Health Prod. 2011;43(5):975-982. doi:10.1007/s11250-011-9794-4

35. Kubuafor DK, Awumbila B, Akanmori BD. Seroprevalence of brucellosis in cattle and humans in the Akwapim-South district of Ghana: public health implications. Acta Trop. 2000;76(1):45-48. doi:10.1016/S0001-706X(00)00088-7

36. Al-majali AM, Talafha AQ, Ababneh MM, Ababneh MM. Veterinary science seroprevalence and risk factors for bovine brucellosis in Jordan. J Vet Sci. 2009;10(1):61-65. doi:10.4142/jvs.2009.10.1.61

37. Alonso S, Dohoo I, Lindahl J, Verdugo C, Akuku I, Grace D. Prevalence of tuberculosis, brucellosis and trypanosomiasis in cattle in Tanzania: a systematic review and meta-analysis. Anim Health Res Rev. 2016;17(1):16-27. doi:10.1017/S146625231600013X

38. Barman NN, Patil SS, Kurli R, Deka P, Bora DP, Deka G. Metaanalysis of the prevalence of livestock diseases in North Eastern Region of India. Vet World. 2020;13.

39. Mangi MH, Kamboh AA, Rind R, et al. Seroprevalence of brucellosis in holstein-friesian and indigenous cattle breeds of Sindh province, Pakistan. J Anim Health Prod. 2015;3(4):82-87. doi:10.14737/journal.jahp/2015/3.4.82.87

\section{Publish your work in this journal}

Veterinary Medicine: Research and Reports is an international, peerreviewed, open access journal publishing original research, case reports, editorials, reviews and commentaries on all areas of veterinary medicine. The manuscript management system is completely online and includes a very quick and fair peer-review system. Visit http://www.dovepress.com/testimonials.php to read real quotes from published authors. 